\title{
AUTOMORPHISMS OF COMMUTATIVE BANACH ALGEBRAS
}

\author{
B. E. JOHNSON
}

\begin{abstract}
This paper presents a new proof of the theorem of Kamowitz and Scheinberg which states that if $\alpha$ is an element of infinite order of the automorphism group of a commutative semisimple Banach algebra then the spectrum of $\alpha$ contains all complex numbers of absolute value 1 . The proof depends on the fact that the only closed translation invariant subalgebras of $l^{\infty}(-\infty,+\infty)$ (pointwise multiplication) for which the restriction of the shift has a complex number of absolute value 1 in its resolvent set are certain spaces of periodic sequences.
\end{abstract}

Let $\mathfrak{U}$ be a commutative Banach algebra and $\alpha$ an automorphism of $\mathfrak{T}$. In [1] Kamowitz and Scheinberg show that either $\alpha^{n}=\iota$, the identity map on $\mathfrak{U}$, for some positive integer $n$, in which case $\sigma(\alpha)$ is a finite union of subgroups of the circle group $T$, or $T \subseteq \sigma(\alpha)$. In this paper we give an entirely different proof of the same result by showing that if $E$ is an open arc in $T$ and $c \in l^{\infty}\left(=l^{\infty}(Z)\right)$ is such that every element of the translation invariant subalgebra of $l^{\infty}(Z)$ generated by $c$ is in $(S-\lambda I)^{3} l^{\infty}$ for all $\lambda$ in $E$, where $S$ is the translation operator, then $c$ is a periodic sequence. This gives the required theorem by considering the sequences $c_{n}=\varphi\left(\alpha^{n} a\right)$, $a \in \mathfrak{U}, \varphi$ an element of the spectrum $\Phi_{\mathfrak{Y}}$ of $\mathfrak{A}$ and $E$ a component of $\boldsymbol{T} \backslash \sigma(\alpha)$.

If $c \in l^{\infty}$ then $\left\{\left(1+n^{2}\right)^{-1} c_{n}\right\} \in l^{1}$ so $c$ is the series of Fourier coefficients of a distribution $\hat{c}=\left(1-D^{2}\right) \sum\left(1+n^{2}\right)^{-1} c_{n} \omega^{n}$ of order 2 on $\boldsymbol{T}$. We refer the reader to [2], in particular pp. 80-83 for information on distributions. We denote the support of $\hat{c}$ by $\tau(c)$. If $a \in \mathfrak{A}, \varphi \in \Phi_{\mathfrak{I I}}$ then $\tilde{a} \in l^{\infty}$ is the sequence $\tilde{a}_{n}=\varphi\left(\alpha^{n} a\right)$.

Lemma. If $\lambda_{0} \in \boldsymbol{T} \backslash \sigma(\alpha)$ then $\lambda_{0} \in \boldsymbol{T} \backslash \tau(\tilde{a})$ for all $a \in \mathfrak{H}$.

Proof. Let $a \in \mathfrak{A}$. We have $\tilde{a}^{\wedge}=\left(1-D^{2}\right) f$ for some $f \in C(T)$. There is a proper open arc $E$ in $T$ containing $\lambda_{0}$ with $E \cap \sigma(\alpha)=\varnothing$. For each $\lambda$ in $E$ there is $b_{\lambda}$ in $\mathfrak{A}$ with $(\alpha-\lambda l)^{3} b_{\lambda}=a$ so putting $\tilde{b}_{\lambda}^{\wedge}=\left(1-D^{2}\right) g_{\lambda}, g_{\lambda} \in C(T)$

Received by the editors July 17, 1972.

AMS (MOS) subject classifications (1970). Primary 46H05; Secondary 46F10, 47C05. 
we get $\left(1-D^{2}\right) f=p_{\lambda}^{3}\left(1-D^{2}\right) g_{\lambda}$ where $p_{\lambda}(\omega)=\omega-\lambda$ so that on $E$

$$
\begin{aligned}
D^{2} f_{0} & =\left(1-D^{2}\right) f=p_{\lambda}^{3} g_{\lambda}-\left(2 D^{2} p_{\lambda}^{3}\right) g_{\lambda}+6 D\left(g_{\lambda} p_{\lambda}^{\prime} p_{\lambda}^{2}\right)-D^{2}\left(p_{\lambda}^{3} g_{\lambda}\right) \\
& =D^{2}\left(p_{\lambda}^{3} h_{\lambda}\right)
\end{aligned}
$$

for some $h_{\lambda} \in C(E), f_{0} \in C(E)$.

Let $E^{\prime}$ be an interval in $\boldsymbol{R}$ which is mapped one-to-one onto $E$ by $x \mapsto e^{i x}$ and let $F_{0}, H_{\lambda}$ etc. be the functions on $E^{\prime}$ corresponding to $f_{0}, h_{\lambda}$ etc. Then $D^{2} F_{0}=D^{2} P_{\lambda}^{3} H_{\lambda}$ in the sense of distributions so that there are complex numbers $k_{\lambda}, l_{\lambda}$ with

$$
F_{0}(y)=P_{\lambda}^{3}(y) H_{\lambda}(y)+k_{\lambda} y+l_{\lambda}
$$

for all $y \in E^{\prime}$. Thus if $e^{i x}=\lambda$ we have $F_{0}(x-h)-2 F_{0}(x)+F_{0}(x+h)=o\left(h^{2}\right)$ as $h \rightarrow 0$ and so the second symmetric derivative of $F_{0}$ is 0 at each point of $E^{\prime}$. From this it follows [3, p. 23, Theorem 10.7] (I am indebted to Professor T. M. Flett for this reference) that $F_{0}$ and $-F_{0}$ are both convex so that $F_{0}$ is linear in $E^{\prime}, D^{2} f_{0}=0$ in $E$ and so $\tau(\tilde{a}) \subseteq \boldsymbol{T} \backslash E$.

THEOREM. If $T \backslash \sigma(\alpha) \neq \varnothing$ then $\sigma(\alpha)$ is a finite union of finite subgroups of $T$.

Proof. If $\lambda_{0} \in T \backslash \sigma(a)$ and $E$ is an open arc in $T \backslash \sigma(a)$ containing $\lambda_{0}$ then, by the Lemma, $E \cap \tau(\tilde{a})=\varnothing$ for all $a$ in $\mathfrak{A}$. Let $A$ be the sup norm closure of $\tilde{\mathfrak{A}}$ in $l^{\infty}$. By the semicontinuity of $\tau$ on $l^{\infty}, A$ is a closed translation invariant subalgebra of $l^{\infty}$ with $E \cap \tau(c)=\varnothing$ for each $c$ in $A$. Put $T=$ $(\bigcup\{\tau(c) ; c \in A\})^{-}$, then $E \cap T=\varnothing$. If $\lambda \in T, n \in Z^{+}$and $J$ is an open interval in $T$ containing $\lambda^{n}$ then we can find an interval $I$ containing $\lambda$ with $I^{n} \subset J$, an element $c$ of $A$ with $\tau(c) \cap I \neq \varnothing$ and an element $d$ of $l^{1}$ with $\hat{d} \in \mathscr{D}(T)$, support $\hat{d} \subset I$ and such that $\hat{d} \hat{c}=(d * c)^{\wedge} \neq 0$. As $A$ is a closed translation invariant subalgebra, $d * c \in A, d * c \neq 0$ so $(d * c)^{n} \in A$, $\tau\left[(d * c)^{n}\right] \subset J$ and $(d * c)^{n} \neq 0$. Thus $\lambda^{n} \in T$.

Let $p$ be an integer greater than $2 \pi$ (length of $E)^{-1}$. As $T \cap E=\varnothing$ and $\lambda$ in $T$ implies $\lambda^{n}$ is in $T, n=1,2, \cdots$, we see that every element of $T$ is an $n$th root of unity for some $n \leqq p$. Thus $T$ is a subset of the set of $p$ ! roots of unity. Let $c \in A$. As in the Lemma, $\hat{c}=\left(1-D^{2}\right) f$ with $f \in C(T)$. Because $\tau(c)$ is finite $f$ is of the form $f(\omega)=r \omega+s \bar{\omega}$ on each interval of $\boldsymbol{T} \backslash \tau(c), \hat{c}$ is a combination of $\delta$ functions at the points of $\tau(c)$ and so $c$ is a periodic sequence with period dividing $p$ !. Thus for all $\varphi$ in $\Phi_{\mathfrak{A}}$, $a$ in $\mathfrak{U}$ we have $\varphi\left(\alpha^{p !} a\right)=\varphi(a)$ which shows $\alpha^{p !}=\iota$ and $\sigma(\alpha)$ consists only of $p$ ! roots of unity.

As $\sigma(\alpha)$ is finite each point is an eigenvalue. If $\alpha(a)=\lambda a, a \neq 0$ then $\alpha\left(a^{n}\right)=\lambda^{n} a^{n}$ where, as $\mathfrak{A}$ is semisimple, $a^{n} \neq 0$ so that if $\lambda \in \sigma(\alpha), n \in Z^{+}$ then $\lambda^{n} \in \sigma(\alpha)$. It follows from this that if $\sigma(\alpha)$ contains one primitive 
$m$ th root of unity it contains all $m$ th roots of unity and so is a finite union of finite subgroups of $\boldsymbol{T}$.

The following result, due to Singer and Wermer, is a corollary of the theorem of Kamowitz and Scheinberg.

COROllary. Let $D$ be a continuous derivation on the commutative Banach algebra $\mathfrak{B}$. Then $D \mathfrak{B} \subseteq$ radical of $\mathfrak{B}$.

Proof. Replacing $D$ by $t D$ if necessary $(0<t<1)$, we can assume $\|D\|<\frac{1}{2}$. Then $\beta=e^{D}$ is an automorphism of $\mathfrak{B}$ with $\|\iota-\beta\|<e^{1 / 2}-1<1$. Put $\mathfrak{U}=\mathfrak{B} / \mathrm{rad} \mathfrak{B}$. Because $\operatorname{rad} \mathfrak{B}$ is invariant under $\beta, \beta$ induces an automorphism $\alpha$ of $\mathfrak{A}$ with $\|\iota-\alpha\|<1$ and hence $\sigma(\alpha) \subseteq\{z: z \in C,|z-1|<1\}$. Thus in the second last paragraph of the proof of the theorem we have length of $E>\pi$ and can take $p=1$, giving $\alpha=\iota$. Thus $\iota-\beta$ maps $\mathfrak{B}$ into $\operatorname{rad} \mathfrak{B}$ and hence so does $D=\log \beta=-\sum n^{-1}(\iota-\beta)^{n}$.

\section{REFERENCES}

1. H. Kamowitz and S. Scheinberg, The spectrum of automorphisms of Banachalgebras, J. Functional Analysis 4 (1969), 268-276. MR 40 \#3316.

2. L. Schwartz, Théorie des distributions, Tome II, Actualités Sci. Indust., 1122, Hermann, Paris, 1951. MR 12, 833.

3. A. Zygmund, Trigonometrical series. Vol. I, 2nd ed. reprinted with corrections and some additions, Cambridge Univ. Press, New York, 1968. MR 38 \#4882.

Department of Mathematics, University of Newcastle Upon Tyne, Newcastle UPON TYNE, ENGLAND 